\title{
Associação entre possível bruxismo e a prática de atividade física em adolescentes escolares: estudo exploratório
}

\author{
Association between possible bruxism and the practice of physical activity in school \\ adolescents: exploratory study
}

Asociación entre posible bruxismo y la práctica de la actividad física en adolescentes escolares: estudio exploratorio

Breno lago Sena de Albuquerque Silva ${ }^{1}$, Laís Lavínia Cruz Soares ${ }^{1}$, Jakelline Cipriano dos Santos Raposo ${ }^{1 *}$, Valdenice Aparecida de Menezes ${ }^{1}$, Viviane Colares ${ }^{1}$, Carolina da Franca ${ }^{1}$, Fabiana de Godoy ${ }^{1}$.

\section{RESUMO}

Objetivo: Verificar a associação entre o possível bruxismo e a prática de atividade física em adolescentes escolares. Métodos: Trata-se de um estudo exploratório, descritivo e analítico de base escolar. Foram incluídos 217 estudantes, na faixa etária de 14 a 19 anos, de ambos os sexos, regularmente matriculados no ensino médio da rede estadual de ensino de uma cidade de Pernambuco. As variáveis avaliadas incluíram os dados sociodemográficos, questões relativas à atividade física e dados relativos ao possível bruxismo que foram retiradas de 3 questionários PeNSE, YRBS e RDC/TMD. Os dados foram analisados utilizando o software STATA 12.0 para Windows. O estudo foi aprovado por Comitê de Ética em Pesquisa. Resultados: Dentre os pesquisados $32,7 \%$ eram bruxistas (sono e vigília), 50,2\% eram sedentários, $61,3 \%$ tinham entre 14 e 16 anos e 64,5\% eram do sexo feminino. Não houve associação estatisticamente significativa em nenhuma das variáveis analisadas em relação ao bruxismo. Houve associação entre o sexo e atividade física (p-valor 0,013 ), relacionando o sexo feminino ao sedentarismo. Conclusão: Não foi observada associação entre o possível bruxismo e atividade física na população estudada.

Palavras-chave: Adolescente, Bruxismo, Exercício físico.

\begin{abstract}
Objective: To analyze the association between bruxism and the practice of physical activity in adolescent students. Methods: This was an exploratory, descriptive and analytical school-based study. We included were 217 students, aged 14 to 19 years, of both genders, regularly enrolled in high school in the state school network of a town in Pernambuco. The variables evaluated included sociodemographic data, questions regarding physical activity, and data regarding possible bruxism that was taken from 3 questionnaires PeNSE, YRBS, and RDC/TMD. The data were analyzed using STATA 12.0 for Windows software. The study was approved by the Research Ethics Committee. Results: Among those surveyed, 32.7\% were bruxists (sleep and wakefulness), $50.2 \%$ were sedentary, $61.3 \%$ were between 14 and 16 years old, and $64.5 \%$ were female. There was no statistically significant association in any of the variables analyzed about bruxism. There was an association between gender and physical activity ( $p$-value 0.013 ), relating females to a sedentary lifestyle. Conclusion: There was no association between bruxism and physical activity in the studied population.
\end{abstract}

Keywords: Adolescent, Bruxism, Exercise.

${ }^{1}$ Universidade de Pernambuco, Recife - PE. *E-mail: jakelline.cipriano@upe.br

SUBMETIDO EM: 12/2021

ACEITO EM: 1/2022

PUBLICADO EM: 2/2022 


\section{RESUMEN}

Objetivo: Verificar la asociación entre el posible bruxismo y la práctica de actividad física en adolescentes escolares. Métodos: Se trata de un estudio exploratorio, descriptivo y analítico basado en la escuela. Se incluyeron 217 estudiantes, de 14 a 19 años, de ambos sexos, matriculados regularmente en la escuela secundaria en la red estatal de educación de una ciudad de Pernambuco. Las variables evaluadas incluyeron datos socio demográficos, preguntas sobre la actividad física y datos sobre el posible bruxismo que se tomaron de 3 cuestionarios PeNSE, YRBS y RDC/TMD. Los datos se analizaron con el programa STATA 12.0 para Windows. El estudio fue aprobado por el Comité de Ética en Investigación. Resultados: Entre los encuestados, el $32,7 \%$ eran bruxistas (de sueño y de vigilia), el $50,2 \%$ eran sedentarios, el $61,3 \%$ tenían entre 14 y 16 años y el 64,5\% eran mujeres. No hubo asociación estadísticamente significativa en ninguna de las variables analizadas en relación con el bruxismo. Hubo una asociación entre el género y la actividad física (valor $p$ 0,013), relacionando el género femenino con el sedentarismo. Conclusión: No se observó ninguna asociación entre el posible bruxismo y la actividad física en la población estudiada.

Palabras clave: Adolescente, Bruxismo, Ejercicio físico.

\section{INTRODUÇÃO}

A adolescência é uma fase de difícil compreensão e bastante dinâmica dentro do período da vida humana, repercutindo de forma direta na formação da personalidade e na inserção no contexto social, sendo uma fase da vida de relevantes mudanças e incertezas com relação ao futuro. Assumir novos papéis, questões como a formação de uma nova identidade, entre outras situações, geram no adolescente uma grande ansiedade, o surgimento de sintomas de depressão e isolamento social, requerendo, assim, apoio daqueles que o cercam (VALLE L e MATTOS M, 2011).

Mudanças globais sem precedentes estão moldando a saúde e o bem-estar dessa geração, de forma que a mobilidade populacional, as comunicações globais, o desenvolvimento econômico e a sustentabilidade dos ecossistemas estão definindo o rumo do futuro de suas vidas. Entre as tendências globais, podemos destacar aquelas que incluem estilos de vida, mercadorias não saudáveis, aumento do comportamento sedentário e outros fatores que representam grandes ameaças à saúde e ao bem-estar dos adolescentes (BLAKEMORE $S$ e MILLS KL, 2014). Esse período da adolescência é marcado por profundas transformações, tanto físicas quanto psicológicas, e tal fato pode favorecer o aparecimento de hábitos parafuncionais, que se excederem o limite fisiológico tolerável, é possível de acarretar agressões ao sistema estomatognático (NAVARRO G, et al., 2018).

O bruxismo pode ser visto como uma parafunção (NAVARRO G, et al., 2018), mas o consenso mais atual o define como sendo um distúrbio do movimento, regulado, principalmente, pelo sistema nervoso central, e não pelo periférico, não sendo causado por fatores anatômicos, como características da oclusão e articulação temporomandibular (LOBBEZOO F, et al., 2018). Também pode estar presente sem necessariamente ter o contato dentário, ocorrendo apenas a tensão dos músculos mastigatórios (LOBBEZOO F, et al., 2018). Por muitas vezes é subestimado, porque esse hábito costuma ser realizado inconscientemente, e a maioria das pessoas desconhece sua presença. Tem uma etiologia multifatorial, mediada centralmente e onde intervêm e interagem fatores fisiopatológicos, morfológicos e psicossociais (SOUSA D, 2017).

Quando se pensa em saúde física e mental, a atividade física (AF) é de extrema importância, pois a literatura demostra uma redução significativa dos sintomas de ansiedade e de estresse, tendo efeito protetor contra transtornos de ansiedade em populações clínicas e não clínicas. Os mecanismos pelos quais o exercício produz esses efeitos provavelmente envolvem uma combinação de fatores biológicos e psicológicos (VORKAPIC-FERREIRA C, et al., 2017; MICHELI L, et al., 2018; MAZYARKIN Z, et al., 2019; KRUK J, et al., 2020). O exercício físico ainda atua mantendo o padrão de secreção de cortisol, que é um hormônio importante usado como biomarcador do estresse psicológico, e essa sensibilidade ao estresse, avaliada pelo cortisol salivar, pode ser um fator psicológico associado ao bruxismo (RAMOS S, et al., 2019; KANDOLA A e STUBBS B, 2020). 
Esse nível de estresse é um fator psicológico muito importante associado ao bruxismo (SALGUEIRO M, 2020). Nesse contexto, o objetivo do presente estudo foi verificar a associação entre o bruxismo e a prática de atividade física em adolescentes escolares de uma cidade de Pernambuco.

\section{MÉTODOS}

Trata-se de um estudo exploratório, transversal e de base escolar realizado em uma cidade do estado de Pernambuco, Brasil. O estudo faz parte de um estudo maior intitulado "Saúde bucal e comportamentos de risco modificáveis na adolescência - acompanhar para prevenir". Os dados do presente estudo foram coletados em um município brasileiro localizado na Região Metropolitana do Recife/PE.

A amostra correspondeu a $10 \%$ da população referente ao estudo maior, sendo obtido o valor de 217 adolescentes. Os critérios de inclusão para compor a amostra foram: estudantes de ambos os sexos, na faixa etária de 14 a 19 anos e regularmente matriculados no ensino médio das escolas públicas estaduais de Olinda-Pernambuco. Os critérios de exclusão foram: estudantes com alguma deficiência ou disfunção que impossibilitasse $o$ autopreenchimento do questionário, foram consideradas perdas o não preenchimento das questões referentes aos dados sociodemográficos, as questões referentes a atividade física, e ao bruxismo.

Foram utilizados três questionários para a coleta de dados. As questões com variáveis independentes relacionadas aos dados sociodemográficos foram retiradas do questionário da Pesquisa Nacional de Saúde do Escolar (PeNSE), sendo elas: a) Qual seu sexo? 1) Feminino; 2) Masculino; b) Qual a sua idade? Categorizada em: 1) 14 - 16 anos; 2) 17 - 19 anos. Os dados relativos à presença do bruxismo foram coletados por meio do questionário Critérios Diagnósticos de Pesquisa em Disfunção Temporomandibular (RDC/TMD), as três perguntas foram: a) Você já percebeu ou alguém falou que você range (ringi) ou aperta os dentes quando está dormindo? b) Durante o dia, você range (ringi) ou aperta os seus dentes? c) Você sente a sua mandíbula (queixo) "cansada" ou dolorida quando acorda pela manhã? Sendo considerado bruxista quem respondeu "sim" a pelo menos uma dessas três questões.

Para avaliar a atividade física foi utilizado a versão traduzida e validada, para o português do Brasil, do questionário Youth Risk Behavior Survey (YRBS), com a seguinte pergunta: "a) Durante os últimos 7 dias, em quantos dias você foi ativo fisicamente por pelo menos 60 minutos por dia? (Considere o tempo que você gastou em qualquer tipo de atividade física que aumentou sua frequência cardíaca e fez com que sua respiração ficasse mais rápida por algum tempo)." Com as opções: (A) Nenhum dia (B) 1 dia (C) 2 dias (D) 3 dias (E) 4 dias (F) 5 dias (G) 6 dias (H) 7 dias. (GUEDES DP e LOPES CC, 2010). Foi considerado sedentário, quem referiu nenhum dia de atividade física; pouco ativo, quem referiu de 1 a 2 dias de atividade física; e fisicamente ativo quem relatou acima de 3 dias.

Os dados foram digitados em dupla entrada no Epidata 3.3 e os erros encontrados na validação foram corrigidos. Para análise de dados foi utilizado o software STATA 12.0 para Windows. Para avaliar o perfil dos alunos foram calculadas as frequências absoluta e relativa. Foi aplicado o teste qui-quadrado para verificar a existência de associação para as variáveis categóricas. Todas as conclusões foram tiradas considerando o nível de significância de $5 \%$.

Este projeto foi desenvolvido de acordo com a resolução no 466/2012, do Conselho Nacional de Saúde do Ministério da Saúde e foi aprovado pelo Comitê de Ética em Pesquisa (CAAE 76609817.1.0000.5207), com a anuência da secretaria de educação do estado de Pernambuco. Os adolescentes maiores de 18 anos convidados a participar desse estudo assinaram Termo de Consentimento Livre e Esclarecido (TCLE) e os menores de 18 anos obtiveram a participação no estudo através da autorização dos pais ou responsáveis por meio do TCLE e assinaram o Termo de Assentimento (TALE) afirmando que gostariam de participar da pesquisa.

\section{RESULTADOS}

As características gerais dos adolescentes incluídos no presente estudo estão apresentadas na Tabela 1. Foi possível observar que a maioria dos adolescentes participantes do estudo estavam na faixa etária de 14 a 16 anos, era do sexo feminino, sedentários e pouco mais de um terço dos escolares eram possíveis bruxistas. 
Tabela 1 - Características sociodemográficas dos adolescentes incluídos no estudo, n=217.

\begin{tabular}{lll}
\hline Variáveis & $\mathbf{n}$ & $\%$ \\
\hline Idade (anos) & & \\
\hline 14 a 16 & 133 & 61,3 \\
17 a 19 & 84 & 38,7 \\
\hline Sexo & & \\
\hline Feminino & 140 & 64,5 \\
Masculino & 77 & 35,5 \\
\hline Atividade Física & & \\
\hline Sedentário & 109 & 50,2 \\
Pouco ativo & 51 & 23,5 \\
Fisicamente ativo & 57 & 26,3 \\
\hline Possível Bruxismo & & \\
\hline Sim & 71 & 32,7 \\
Não & 146 & 67,3 \\
\hline
\end{tabular}

Fonte: Silva BISA, et al., 2021.

A Tabela 2 apresenta as associações entre possível bruxismo e as variáveis independentes da pesquisa. Dentre as estudadas, não houve associação estatisticamente significativa.

Tabela 2 - Associação entre características sociodemográficas, atividade física e bruxismo.

\begin{tabular}{|c|c|c|c|}
\hline \multirow[b]{2}{*}{ Variáveis } & \multicolumn{2}{|c|}{ Possível Bruxismo } & \multirow[b]{2}{*}{ p-valor } \\
\hline & $\begin{array}{c}\text { Sim } \\
\text { (\%) }\end{array}$ & $\begin{array}{c}\text { Não } \\
\text { n (\%) }\end{array}$ & \\
\hline \multicolumn{4}{|l|}{ Idade (anos) } \\
\hline 14 a 16 & $43(32,3)$ & $90(67,7)$ & 0,878 \\
\hline 17 a 19 & $28(33,3)$ & $56(66,7)$ & \\
\hline \multicolumn{4}{|l|}{ Sexo } \\
\hline Feminino & $51(36,4)$ & $89(63,6)$ & 0,116 \\
\hline Masculino & $20(26,0)$ & $57(74,0)$ & \\
\hline \multicolumn{4}{|l|}{ Atividade Física } \\
\hline Sedentário & $36(33,0)$ & $73(67,0)$ & 0,829 \\
\hline Pouco ativo & $18(35,3)$ & $33(64,7)$ & \\
\hline Fisicamente ativo & $17(29,8)$ & $40(70,2)$ & \\
\hline
\end{tabular}

Legenda: $\left.{ }^{*}\right)$ Teste qui-quadrado

Fonte: Silva BISA, et al., 2021.

\section{DISCUSSÃO}

O estudo mostrou que um terço da população estudada apresentou possível bruxismo. Os valores relatados em outros estudos sobre bruxismo em adolescentes trazem uma ampla variação, de $5,3 \%$ até 47,5\%. (ARMAN K, et al., 2016; CARRA MC, et al., 2011; MURRIETA J, et al., 2014; PERLMAN E, et al., 2016; PRADO I, et al., 2018; TÜRKOĞLU S, et al., 2013). Essa discrepância de valores possivelmente se dá pela ampla variação de faixas etárias empregadas, diferentes critérios utilizados para diagnosticar possível bruxismo, não especificação do tipo de bruxismo (do sono ou de vigília) e o número da amostra (FIRMANI M, et al., 2015). Outro ponto que deve ser levado em consideração é o perfil da população estudada. Além disso, muitos estudos não distinguem corretamente as faixas etárias, sendo encontrados crianças e adolescentes e em alguns casos até adultos jovens no mesmo estudo o que dificulta a análise, porque existem diferenças fisiológicas entre esses grupos e quando não descrito na pesquisa, pode confundir os resultados.

Perlman E, et al. (2016), salienta a dificuldade no diagnóstico do bruxismo de vigília apenas pela aplicação de um questionário, assim a adição de exame clínico poderia resultar em um desfecho diferente para 
pesquisa. Em seu estudo, as opções de resposta foram limitadas a 'Não', 'Sim' e 'Desconhecido', que podem ter introduzido viés de resposta. Assim, uma resposta positiva pode fornecer informações falsas e, definitivamente, não aborda a verdadeira situação epidemiológica. Essa questão deve ser sempre levada em consideração em leitura de artigos que tratam de bruxismo autorreferido.

Com a intenção de resultados mais fidedignos, Gastañaga VAA, et al. (2020), em seu estudo usou a seguinte classificação: Possível bruxismo: quando confirmado por um autorrelato baseado no questionário e/ou a anamnese; Provável bruxismo, quando baseado no autorrelato e em exame clínico; e Definitivo, quando confirmado com o autorrelato, o exame clínico e polissonografia. Os mesmos defendem que o método padrão ouro para o diagnóstico do bruxismo do sono é o uso da polissonografia, pois fornece registros do sono e inclui eletroencefalograma, eletromiograma, registros e sinais de eletrocardiograma e gravações simultâneas de áudio e vídeo. No entanto, suas desvantagens são o custo, o tempo necessário para a avaliação e a mudança no ambiente que pode influenciar o comportamento real (GASTAÑAGA VAA, et al., 2020).

Ao comparar os estudos de Perlman E, et al. (2016) e Gastañaga VAA, et al. (2020), é importante enfatizar que os estudos selecionados estão em diferentes contextos. Uma situação refere-se ao local onde os estudos foram realizados, encontrando diferentes prevalências em diferentes países (MACHADO E, et al., 2014). A exemplo, tem-se o estudo de Perlman E, et al. (2016), que foi realizado em Israel, em um momento em que a sociedade local sofria maior pressão psicológica pela contínua ameaça à sua segurança e tensão econômica, encontrando uma prevalência de 19,2\% de bruxismo de vigília, considerando o contexto estressante em que vivem os adolescentes israelenses, os níveis de sintomas psicológicos e físicos eram comumente relatados.

Entretanto a pesquisa realizada por Murrieta J, et al. (2014) com adolescentes mexicanos demonstrou que $47,5 \%$ dos entrevistados relataram hábitos bruxistas, podendo ser influenciado pela situação econômica que a América Latina vivia na época. Fato esse, que ratifica a importância em compreender a população estudada, para entender e interpretar os desfechos relacionados às pesquisas (ANJOS R, et al., 2020).

Quanto a faixa etária, o possível bruxismo não apresentou diferença estatisticamente significativa ( $p$-valor 0,878). Perlman E, et al. (2016), dividiram em faixas etárias entre 12-15 anos e 16-18 anos e, no bruxismo de vigília, assim como neste estudo, não encontraram associação estatisticamente significativa. O que pode ser justificado pelos grupos das duas pesquisas serem divididos quase que de maneira semelhante. Os resultados de Wetselaar $P$, et al. (2021) revelam um aumento na prevalência de bruxismo do sono com o aumento da idade. Vale salientar, que a faixa etária do que é considerada adolescência difere. O estudo analisou adolescentes holandeses em apenas duas idades: 17 e 23 anos, e por utilizar uma população com faixa etária mais avançada que o usual, o autor justificou a diferença de idade pelo aumento da atividade da musculatura mastigatória, ou pelo fato dos adolescentes dessa idade muitas vezes terem um companheiro de cama que pode relatar seu ranger durante o sono.

Carra MC, et al. (2011) dividiu sua faixa etária geral em duas, sendo de 7-12 anos e 13-17 anos, notando uma prevalência maior no segundo grupo. Em contrapartida, Firmani M, et al. (2015) achou maior prevalência nos adolescentes mais novos, justificando que essa redução da prevalência na adolescência pode ser influenciada pela subnotificação, uma vez que nos adolescentes mais velhos, os pais tendem a não dormir tão perto de seus filhos, consequentemente, não percebem possíveis alterações em seus padrões de sono.

Quanto ao sexo, este estudo não apresentou diferença significativa, corroborando com os estudos de Restrepo C, et al. (2009), Türkoğlu S, et al. (2013), Manfredini D, et al. (2013) e Firmani M, et al. (2015), que não encontraram diferença estatisticamente significativa do possível bruxismo entre meninos e meninas. Entretanto, Melo Junior (2019) e Magalhães B, et al. (2014) em seus estudos, atribuíram uma predileção ao sexo feminino, justificando o achado devido a diferenças hormonais. Segundo Mong JA e Cusmano DM (2016), as mudanças na produção de esteroides ovarianos comuns ao período de puberdade e menopausa estão associadas com aumento da prevalência de insônia e pior qualidade no padrão de sono. Pensamento esse, que corrobora com Song YL, et al. (2018), pois defendem que os hormônios femininos desempenham um papel importante na etiologia das parafunções, enquanto os hormônios masculinos parecem ter uma função protetora. 
Este estudo demonstrou que mais da metade dos adolescentes entrevistados foram considerados sedentários. Corroborando com os achados de Santana C, et al. (2021), onde os resultados mostraram altas prevalências de sedentarismo entre escolares brasileiros $(56,3 \%)$, valor semelhante à revisão sistemática de Arundell L, et al. (2016), que descobriram que o comportamento sedentário durante a infância, independentemente dos níveis de atividade física, está aumentando, por exemplo: assistir televisão extensivamente está positivamente associado à composição corporal e diminuição do desempenho acadêmico.

Nesse estudo de Arundell L, et al. (2016), os adolescentes passaram 57\% do seu tempo após a escola em atividades sedentárias. Um período do dia que tem o potencial de contribuir substancialmente para os níveis de comportamento sedentário diário. Esses dados são preocupantes, segundo a Organização Mundial da Saúde (OMS), em crianças e adolescentes, a atividade física proporciona inúmeros benefícios para saúde, entre eles, a melhora da aptidão física, saúde cardiometabólica, "saúde óssea, cognição (desempenho acadêmico e função executiva), saúde mental (redução dos sintomas de depressão) e redução da adiposidade" (CAMARGO EM e AÑEZ CRR, 2020).

Em estudos que usaram a mesma ferramenta de medição e que foram conduzidos em países de baixa e média renda, os resultados são geralmente consistentes (CALEYACHETTY R, et al, 2015; PELTZER K, 2010; RIBEIRO JAB, et al, 2013). Al Subhi LK, et al. (2015), em um estudo cruzado de países, demonstrou que a prevalência geral de inatividade física foi de $81 \%$ em 10 países do leste do Mediterrâneo. Caleyachetty $R$, et al. (2015), em um estudo de meta-análise conduzido em 65 países de baixa e média renda, baixa atividade física foi demonstrada em $71,4 \%$ dos adolescentes. Em oito países africanos, Peltzer K, et al. (2010), descobriram que $85,8 \%$ das crianças em idade escolar eram frequentemente inativas fisicamente.

Esses dados se afastam do estudo de Ribeiro JAB, et al. (2013) na cidade de Pelotas/RS, que achou um valor de $12,5 \%$. Uma hipótese para divergência do resultado apresentado se dá pela diferença de ferramenta usada para medir inatividade física e pela participação da escola em projetos esportivos como, atletismo, basquete, voleibol, futsal. Azevedo Junior MR, et al. (2006) sugerem que a Educação Física, quanto matéria escolar obrigatória, seria capaz de gerar uma gama de ofertas de práticas de atividades físicas, como musculação, ginástica, que pudessem estimular o interesse da população escolar de adolescentes e jovens.

Azevedo Junior MR, et al. (2006) ainda apontam que, quando indivíduos são praticantes de atividade física na adolescência, a probabilidade de se tornarem adultos ativos são maiores e que a adoção de um estilo de vida ativo é um fenômeno multicausal e que pode ser influenciado pela experiência positiva desde cedo. Portanto, o incentivo à prática de atividade física nessa fase, torna-se um fator importante para reduzir os níveis de sedentarismo na vida adulta. Barufaldi L, et al. (2012), realizaram uma revisão sistemática e metaanálise para investigar a prevalência de inatividade física entre adolescentes no Brasil, no entanto, observaram a falta de concordância entre a maioria dos estudos, o resultado foi uma ampla variação de 5,4\% até $91 \%$. Supõe-se que essas diferenças são decorrentes das características da população investigada, da diversidade de instrumentos de coleta utilizados ou dos pontos de corte adotados.

Observou-se que pouco mais de um terço dos participantes eram bruxistas, e também que quase $70 \%$ dos adolescentes participantes que praticavam atividade física, entre pouco ativo e fisicamente ativo, não apresentaram bruxismo, contudo, não houve diferenças estatisticamente significativas, demonstrando a não associação entre essas duas variáveis. A hipótese era que a atividade física fosse um fator protetor para o bruxismo, tendo em vista que, de acordo com Camargo EM e Añez CRR (2018), ela melhora a saúde mental, um dos possíveis fatores associados ao bruxismo, de acordo com Guo H, et al. (2018).

Entretanto, este estudo não pode provar isso, assim como a pesquisa de Navarro G, et al. (2018), também desenvolvida com adolescentes brasileiros e com métodos semelhantes a este estudo, com uso de questionário de autorrelato para o bruxismo e prática de atividade física. Navarro G, et al. (2018) também partiu da premissa que os estudantes sedentários poderiam apresentar mais hábitos parafuncionais, bruxismo do sono e dor extrafacial, pois a prática de atividade física pode diminuir o estresse que piora esses problemas. 
Quanto às limitações da pesquisa, pode-se citar que este foi um estudo transversal, portanto, fez avaliação em uma população bem específica, com os dados mensurados uma única vez e no geral, nesse tipo de trabalho é difícil estabelecer uma relação de causalidade. Apesar de não provar a associação proposta, existe plausibilidade na hipótese, por isso sugere-se que sejam realizados estudos com amostras mais representativas. Outro fator importante foi a forma de avaliação, sugere-se que em estudos futuros a atividade física seja categorizada em leve, moderada e vigorosa, pois pode ser que isso influencie nessa relação, com relação aos instrumentos para verificação do bruxismo, os questionários de autorrelato ainda são de mais fácil acesso para estudos epidemiológicos (PRASSAD S, et al., 2021).

\section{CONCLUSÃO}

De acordo com este estudo, concluiu-se que o possível bruxismo esteve presente em $32,7 \%$ dos adolescentes e não apresentou associação estatisticamente significante com nenhuma das variáveis analisadas. Em relação a atividade física, $50,2 \%$ dos adolescentes eram sedentários, sendo mais prevalente no sexo feminino. Atividade física não apresentou diferença estatisticamente significante quanto a faixa etária. Em relação ao possível bruxismo e atividade física não foi possível observar uma associação estatisticamente significante na população estudada, destacando a importância de mais estudos, com amostra maior, longitudinais e de intervenção a respeito do tema.

\section{AGRADECIMENTOS}

Agradecemos o apoio da Universidade de Pernambuco, da Secretaria de Educação de Pernambuco e dos/as gestores/as das escolas que viabilizaram essa pesquisa, assim como aos/as adolescentes participantes.

\section{REFERÊNCIAS}

1. AL SUBHI LK, et al. Prevalence of physically active and sedentary adolescents in 10 Eastern Mediterranean countries and its relation with age, sex, and body mass index. Journal of physical activity \& health, 2015; 12(2): $257-265$.

2. ANJOS R, et al. Bruxismo associado a fatores mentais em adolescentes: uma revisão integrativa. Research, Society and Development, 2020; 9(9).

3. ARMAN K, et al. Stress experience and effect on self-perceived oral health status among high school students. Stomatologija. Baltic Dental and Maxillofacial Journal, 2016; 18: 75-9.

4. ARUNDELL L, et al. A systematic review of the prevalence of sedentary behavior during the after-school period among children aged 5-18 years. International Journal of Behavioral Nutrition and Physical Activity, 2016; 13(1): 1-9.

5. AZEVEDO JUNIOR MR, et al. Atividades físicas e esportivas na adolescência: mudanças de preferências ao longo das últimas décadas. Revista Brasileira de Educação Física, 2006; 20(1): 51-58.

6. BARUFALDI L, et al. Meta-analysis of the prevalence of physical inactivity among Brazilian adolescents. Cadernos de Saúde Pública, 2012; 28(6): 1019-1032.

7. BLAKEMORE S, MILLS KL. Is Adolescence a Sensitive Period for Sociocultural Processing?. Annual Review of Psychology, 2014; 65(1): 187-207.

8. CALEYACHETTY R, et al. Prevalence of behavioural risk factors for cardiovascular disease in adolescents in lowincome and middle-income countries: an individual participant data meta-analysis. The Lancet Diabetes \& Endocrinology, 2015; 3(7): 535-544.

9. CAMARGO EM, AÑEZ CRR. Diretrizes da OMS para atividade física e comportamento sedentário: num piscar de olhos. Genebra: Organização Mundial da Saúde, 2020.

10. CARRA MC, et al. Prevalence and risk factors of sleep bruxism and waketime tooth clenching in a 7- to 17-yr-old population. European Journal of Oral Sciences, 2011; 119: 386-394.

11. FIRMANI M, et al. Sleep bruxism in children and adolescents. Revista chilena de pediatría, 2015; 86(5): 373-9.

12. GASTAÑAGA VAA, et al. Bruxismo en niños y adolescentes: Revisión de la literatura. Odovtos: International Journal of Dental Sciences, 2020; 22(1): 9.

13. GUEDES DP, LOPES CC. Validação da versão brasileira do Youth Risk Behavior Survey 2007. Revista de Saúde Pública, 2010; 44(5): 840-50.

14. GUO H, et al. The risk factors related to bruxism in children: A systematic review and meta-analysis. Archives of oral biology, 2018; 86: 18-34. 
15. KANDOLA A, STUBBS B. Exercise and Anxiety. Advances in experimental medicine and biology, 2020; 1228: 345352.

16. KRUK J, et al. Physical exercise and catecholamines response: benefits and health risk: possible mechanisms. Free Radical Research, 2020; 54(2-3): 105-125.

17. MACHADO E, et al. Prevalence of sleep bruxism in children: A systematic review, Dental Press Journal of Orthodontics, $2014 ; 19(6): 54-61$.

18. MAGALHÃES B, et al. Temporomandibular disorder: otologic implications and its relationship to sleep bruxism. Brazilian journal of otorhinolaryngology, 2018; 84: 614-619.

19. MANFREDINI D, et al. Epidemiology of bruxism in adults: a systematic review of the literature. Journal of orofacial pain, 2013; 27(2): 99-110.

20. MAZYARKIN Z, et al. Health benefits of a physical exercise program for inpatients with mental health; a pilot study. Journal of Psychiatric Research, 2019; 113: 10-16.

21. MELO JÚNIOR PC. Prevalência das Desordens Temporomandibulares e análise de fatores de risco em um grupo de adolescentes brasileiros. Tese (Doutorado em Odontologia) - Faculdade de Odontologia de Pernambuco. Universidade de Pernambuco, Camaragibe, 2019; 63 p.

22. MICHELI L, et al. Depression and adult neurogenesis: Positive effects of the antidepressant fluoxetine and of physical exercise. Brain Research Bulletin, 2018; 143: 181-193.

23. MONG JA, CUSMANO DM. Sex differences in sleep: Impact of biological sex and sex steroids. Philosophical Transactions of the Royal Society B: Biological Sciences, 2016; 371(1688).

24. MURRIETA J, et al. Bruxism frequency and dental occlusion type in a group of Mexican adolescents. Journal of oral research, 2014; 3(4): 211-217.

25. NAVARRO G, et al. Parafunctional habits and its association with the level of physical activity in adolescents. Brazilian Journal of Pain, 2018; 1(1): 46-50.

26. PELTZER, K. Leisure Time Physical Activity and Sedentary Behavior and Substance Use Among In-School Adolescents in Eight African Countries. International Journal of Behavioral Medicine, 2010; 17(4): 271-278.

27. PERLMAN E, et al. Self-Reported bruxism and associated factors in Israeli adolescents. Journal of Oral Rehabilitation, 2016; 43(6): 443-450.

28. PRADO I, et al. Study of Associated Factors With Probable Sleep Bruxism Among Adolescents. Journal Of Clinical Sleep Medicine, 2018; 14(8): 1369-1376.

29. PRASSAD S, et al. Associations among masticatory muscle activity, physical activity and self-reported oral behaviours in adult women. Clinical Oral Investigations, 2021; 25(8): 5049-5059.

30. RAMOS S, et al. A influência do exercício físico sobre o cortisol e glicose sanguínea de praticantes de atividade física. RBNE-Revista Brasileira De Nutrição Esportiva, 2019; 13(81): 666-674.

31. RESTREPO C, et al. Treatment of bruxism in children: A systematic review. Quintessence International, 2009; $40(10): 849-55$.

32. RIBEIRO JAB, et al. Nível e importância atribuídos a prática de atividade física por estudantes do ensino fundamental de uma escola pública de Pelotas/RS. Revista Mackencie de Educação Física e Esporte, 2013; 12(2):13-25.

33. SALGUEIRO M, et al. Effects of Photobiomodulation in Children with Down Syndrome and Possible Sleep Bruxism: Protocol For A Randomized, Controlled, Blind, Clinical Trial: Study protocol clinical trial (SPIRIT compliant). Medicine, 2020; 99(17): e19904.

34. SANTANA C, et al. Association between parental supervision and sedentary behavior and physical inactivity and among brazilian adolescents. Ciência \& Saúde Coletiva, 2021; 26(2): 569-580.

35. SONG YL, et al. Association between temporomandibular disorders and pubertal development: A systematic review. Journal of Oral Rehabilitation, 2018; 45(12): 1007-1015.

36. SOUSA D. Associação entre o Cronotipo, Bruxismo de Sono Auto - Referido e o Bruxismo de Vigília em Alunos de Medicina Dentária - Estudo Multicêntrico entre Portugal e Brasil. Dissertação (Mestrado Integrado em Medicina Dentária) - Faculdade de Medicina. Universidade de Coimbra, Coimbra, 2017; 58p.

37. TÜRKOĞLU S, et al. Psychiatric disorders and symptoms in children and adolescents with sleep bruxism. Sleep And Breathing, 2013; 18(3): 649-654.

38. VALLE L, MATTOS M. Adolescência: as contradições da idade. Revista Psicopedagogia, 2011; 28(87): $321-323$.

39. VORKAPIC-FERREIRA C, et al. Nascidos para correr: a importância do exercício para a saúde do cérebro. Revista Brasileira de Medicina do Esporte, 2017; 23(6): 495- 503.

40. WETSELAAR P, et al. The prevalence of awake bruxism and sleep bruxism in the Dutch adolescent population. Journal of Oral Rehabilitation, 2021; 48(2): 143-149. 